\title{
Floater survival affects population persistence. The role of prey availability and environmental stochasticity
}

\author{
Vincenzo Penteriani, Fermín Otalora and Miguel Ferrer
}

\begin{abstract}
We develop two individual-based models using a large and detailed data set (information gathered over more than a century) on a population of a longlived and territorial predator, the Spanish imperial eagle. We investigated the relationship between survival and predator pressure, prey behaviour and patch availability (i.e. settlement areas). Survival of dispersing individuals was highly dependent on the number of available settlement areas, mediated by prey availability. Changes in prey behaviour due to predation pressure (e.g. shifting from diurnal to nocturnal activity) can decrease their availability for predators even if the density significantly exceeds the predator needs. Environmental stochasticity had a strong influence on population viability when it occurred in a synchroneous way between breeding and settlement areas, and an increase in floater mortality negatively influenced stability and dynamics of the breeding segment of populations in reproductive areas. Our simulations demonstrated the link between the dynamics in settlement and breeding areas: factors affecting floater survival also influence whole population dynamics. Moreover, model outputs provided insights into the relationship between environmental stochasticity and population dynamics.
\end{abstract}

V. Penteriani and M. Ferrer, Dept of Applied Biology, Estación Biológica de Doñana, CSIC, Avda. María. Luisa s/n, Pabellón del Perú, ES-41013 Seville, Spain (penteriani@ebd.csic.es). - F. Otalora, Laboratorio de Estudios Cristalográ ficos, IACT (CSIC/Univ. of Granada), Campus Fuentenueva, Facultad de Ciencias, ES-18002 Granada, Spain.

Dispersal mechanisms and patterns have been the subject of much study in recent times, leading to a large quantity of theoretical considerations and empirical evidence on several aspects of animal movement (Clobert et al. 2001, Nathan 2001, but also revision in Paradis et al. 2002). Many of these studies focused on individual strategies and factors driving the dispersal process, as well as on how dispersal enables individuals to depart from unfavourable habitat, avoid predation and competition, search for mates, avoid inbreeding and (re)colonise habitats (reviewed by Begon et al. 1996).

However, there is little theory on how interactions among dispersing individuals, food, and patches availability may affect the survival of dispersing individuals and, possibly, the persistence of the whole population (floaters $\neq$ breeders). In fact, the dependence of floater survival on suitable settlement areas (i.e. temporary settling zones used during dispersal), and the effect of mortality during dispersal on breeding populations have in fact received little or no attention (Ruxton et al. 1997, Acosta 2002, Bascompte et al. 2002, Flather and Bevers 2002, Yáber and Rabenold 2002). Dispersal has been usually studied to identify what factors in breeders/ breeding areas determine the observed patterns of movement. More rarely, the focus has been on contrary phenomenon, i.e. the relationship between the nonbreeding segment of the population in the settlement areas (e.g. mortality) and the dynamics of the breeding 
segment in the reproductive areas (Danchin and Cam 2002).

Settlement areas are usually unknown for most species and, for this reason, the behaviour and turnover of individuals within them are poorly studied. Consequently, such zones are usually less protected than breeding territories, which may lead to increased risk of mortality for dispersing individuals. In addition, dispersal of individuals across unfamiliar terrains and losses during dispersal are likely to be considerable. In these areas, habitat destruction and decline in survival rates could be critical factors affecting the persistence of the whole population (especially for threatened species). This may render the species more vulnerable to extinction by demographic and environmental stochasticity (Hill et al. 2002). Despite this, as highlighted by Ruxton et al. (1997), several models assume that dispersal occurs with no cost in terms of increased mortality and, consequently, that the events affecting dispersing individuals have no effect on the stability of populations.

When studying processes characterised by temporally (reproduction vs dispersal) and spatially (breeding vs settlement areas) disjunct locations, we should consider the influence of environmental stochasticity (changes in the physical or biological environment affecting all individuals in a population in a similar way) on the different segments of a population. Consideration should also be given to the possible effects attributable to the synchroneous or asynchroneous way in which these situations arise. Moreover, environmental stochasticity represents an interesting area for theoretical applications in the context of dispersal and population dynamics (Harding and McNamara 2002, Sæther et al. 2002). It is intuitively clear that increased environmental stochasticity should typically have a negative influence on population dynamics due to increased extinction risk, speeding up the extinction process (Alvarez 2001). However, the effects of habitat loss and mortality increase, extinction probability, and environmental stochasticity have been mainly studied on breeding areas, and considered as less important or ignored for settlement areas. In this paper, we present a simulation study concerning two individual-based models built on data obtained through longterm monitoring of a population of a Mediterranean predator, the Spanish imperial eagle Aquila adalberti (Ferrer 2001). The simulations are based on several well studied aspects of the behaviour of radiotagged individuals of this eagle (Ferrer 1992, 1993a,b), allowing us to build the models on precise information about some of the main critical needs in dispersal research (Walters 2000), i.e. how a predator species moves and interacts with the environment. Individual-based models are a powerful approach to the study of complex patterns arising from the interactions of individuals, allowing predictions of the dynamics of real-world populations based on the behaviour of individuals (De Angelis and Gross 1992, Ferrer et al. 2004).

In order to capture the relationships between floater and breeder segments of populations, we built two different models to investigate mainly the effects of: (1) prey accessibility and number of available settlement areas on survival of dispersing individuals, (2) synchroneous/asynchroneous variation of environmental conditions in the breeding and the settlement areas on the size and productivity of both the floater and breeder sectors, and (3) mortality increase by environmental stochasticity in settlement areas on the size and productivity of the breeding population. Such topics are relevant to several of the main questions in dispersal research, such as the use of dispersal data in evaluating alternative models of avian population structure, and in conservation biology, where knowledge of behaviour during dispersal is critical (Walters 2000).

\section{Methods}

The species and the "eagle-rabbit game"

The Spanish imperial eagle is a large (2500-3500 g), sedentary and territorial bird of prey, characterised by: (a) low reproductive rates ( 0.75 chicks per pair per year), (2) an immature phase of 4-5 years, and (c) a longevity of approx. 21-22 years (Ferrer and Calderón 1990). Typically, breeding pairs include two adults, but some pairs can be mixed, that is one or both mates have not attained adult plumage (B5 years old; Ferrer and Bisson 2003). Immature unpaired eagles are not territorial and move among temporary settlement areas during the dispersal period, as is typical of several species of vertebrate predators (Ferrer 1993a, b).

There is a complex relationship between the number of dispersing individuals, the time they spend in each settlement area and the number of available areas. The main factor influencing these components is the time (approx. 12 days) that the rabbit (Oryctolagus cuniculus L.), the main prey of this eagle, takes to change its behaviour (e.g. activity timetable and use of space) under predation pressure. The continuous and predictable presence of this large predator in a small area (settlement areas average approx. $433 \mathrm{ha}$ ) forces the rabbit to temporarily modify its behaviour (i.e. it switches from diurnal to nocturnal activity), consequently decreasing prey availability (Ferrer 1993a, b). The consequent increase in mortality by predation by owls and mammals at night may be the main cause of the rabbit reverting to diurnal habits. This makes rabbit available for eagles again. One of the consequences of this "eagle-rabbit game" is that immature eagles use different temporary settling areas in rotation, the average stay being 12 days (Ferrer 1993a). Different individuals select the same settlement areas contemporaneously. In our opinion, the 
discovery of such a game represents a key component in the understanding of the relationships between a predator species and its main prey. This game is based on the behavioural response of the prey to predation pressure rather than on its density. Such a phenomenon may be widespread in those predator species that depend mainly on a single prey species, whether temporarily or throughout the year, in their whole distribution area or in only a part of their range (e.g. eagle owl Bubo bubo, Iberian lynx Lynx pardinus and Canadian lynx Lynx canadensis; Gamarra and Solé 2000, Penteriani et al. 2002).

\section{Empirical basis of models}

The data set of the Spanish imperial eagle in Doñ ana (southwestern Spain, 37844?N, 3828?O) is, to our knowledge, one of the largest and longest today available on a vertebrate species (data collection started in 1890). Moreover, during approx. fifteen years of research (1986-2000), 60\% of breeding and floater individuals were marked with radio tags (Ferrer 2001). Such information provides a strong basis for broad-spectrum modelling accounting for the general relationship (e.g. whole population persistence, breeding performance) between floaters and breeders of a population of a predator species. Such long term information on one of the most threatened raptors in the world can also be a unique and invaluable source of information for the general understanding of small population dynamics and the building of strong predictive models as a tool for conservation. Therefore, the structure, parameters and initial values of our models were guided and set by both: (a) the observed long term (more than one century) population dynamics, for which previous simulations proved the similarity between modelled patterns and empirical data (Ferrer and Bisson 2003, Ferrer and Penteriani 2003, Ferrer et al. 2004), and (b) the more general information we obtained in our eagle field study on the functioning of a typical Mediterranean predator moving within a heterogeneous landscape and depending on a single main prey.

Characteristics of the model of the ratio "number of individuals: available settlement areas”

The eagle-rabbit game model assumes that: (a) the decrease in hunting success of the eagle, despite increased hunting effort, leads individuals to change food patches and move to another settlement area after approx. 12 days; (b) in a situation characterised by a high number of individuals and few settlement areas (which can be due to increased reproductive success in the breeding population, decreased mortality of dispersing individuals and/or reduction of the number of zones available) prey stress can be prolonged by the arrival of successive floaters in the same area. In this case, the alteration in rabbit behaviour leads to long periods of fasting for the dispersing eagles, which die from starvation after approx. 20 days (Ferrer and Dobado-Berrios 1998).

\section{Settlement areas}

Each settlement area can take any value between 0 and 1, indicating the rabbit state of alert. Because low values indicate high stress, this parameter can also be considered as an index of the possibility that an eagle catches a prey. The probability of successful hunting can be set to a given value or be initialised to a random value, decreasing with the increase in the time an individual spends in this area or increasing each day the area is predator-free. The daily decrease/increase of this probability is set in such a way as to shift from the higher (1) to the lower (0) value in twelve days (approx. the real time during which we detected the alteration of rabbit behaviour). Each area is characterised by the number of individuals present. The model has no limits on the number of either dispersers or settlement areas.

\section{Eagles}

Each individual is characterised by a value varying from 0 to 1 and indicating its health (or nutritional) condition. It can be set to a given value or be initialised to a random value, and represents a direct measure of the bird's status: the lower this value, the more starved the eagle is. Therefore, for each day the eagle spends in the area, the probability of successfully catching a rabbit is a stochastic function of the status of rabbit alert. If this threshold allows the predator to obtain a prey, its health status increases by a fixed amount (foodUnit in the model). However, the eagle condition decreases each day by a fixed amount (dayConsumption). This implies that, when the value indicating the individual condition goes beyond a fixed threshold (starvingAt), the eagle moves to another area randomly selected from all the available ones. When this same value reaches a fixed limit (dyingAt) the eagle dies. For example, for dayConsumption $=0.05$, foodUnit $=3>$ dayConsumption, starvingAt $=0.3$ and dyingAt $=0.1$, eating a rabbit gives an eagle sufficient energy to allow it to survive three days of fasting and an eagle will die after a maximum of 20 days without successful hunting. The eagle-rabbit game for a scenario with 1 eagle and 2 settlement areas over 90 days is represented in Appendix A (description of the formal equations on which the model was built) and Appendix B (graphical representation). 
Types of simulations: fixed and varying number of floaters

We ran two different types of simulations, that we called fixed and varying number of floaters. In the first simulation we tested the model for a specific scenario, i.e. the number of dispersing individuals and available areas were fixed, and the only possible event was the death of the individuals by starvation. One hundred replicates, each five years long, were performed and averaged. The model output consists of number of areas (M), number of individuals (N) and survival (S), expressed as "final population size/original population size", i.e. the proportion of the initial population surviving after 5 years (since mortality only occurs by starvation due to the decreasing probability of catching a prey). At the starting time step $(\mathrm{t}=0)$, there were $\mathrm{M}$ areas (25M519) and $\mathrm{N}$ individuals (15N 519).

The varying number of floater simulation considers that individuals are incorporated as floaters into the original pool of dispersers as a function of productivity in the stable segment of the breeding population. This simulation represents a first step in our investigation of the possible relationships between the dispersing and breeding fractions of a population. At the beginning of the simulation $(t=0)$, the system of settlement areas $(n=10)$ is predator-empty and the number of individuals that are integrated each year is generated as an aleatory variable depending on the annual productivity. Productivity is another parameter of the simulation and ranges from 1 to 39 fledglings year ${ }^{-1}$. This model ran with 10 areas: more areas increased the time of the simulation outputs without giving more information on the process. The output consists of five parameters relating to the following characteristics of floaters: (1) time (the simulation recalculates all status information on a daily basis, but because only one piece of data per month is needed for the analysis, output is restricted to this frequency); (2) incorporations (eagles arriving that month which are incorporated in the system); (3) deaths (eagles that die that month); (4) population (living fraction of the starting number of floaters); and (5) mortality. We only reported the results concerning the variation of these five parameters for five values of productivity: $1,10,20,30$ and 39 fledglings year ${ }^{-1}$.

\section{Effects of synchronous and asynchronous variations in environmental conditions in settlement and breeding areas}

The second model was built to assess the effects of synchronous and asynchronous variations in environmental conditions on dispersers and their possible consequences for the breeding portion of the population. The scenario with synchroneous variation in environ- mental conditions is intended to assess the consequences of floater mortality on the whole population when the effects of time on the quality of the habitat (i.e. good or poor year) are similar in two spatially disjunct areas (breeding vs settlement areas). The scenario with asynchroneous variation of environmental conditions accounts for the opposite situation, i.e. when year has different effects in spatially disjunct areas (e.g. when the settlement area habitat is structurally different from the breeding area or far from breeding territories).

Our models include density-dependent compensation, individual (eagle > eagle and eagle >rabbit) as well as individual $>$ area interactions, because models without density-dependent compensation and species interactions inevitably lead to the demise or explosion of a population, as well as erroneous inferences about a population's responses to individual intra- and interspecific interactions (Sutherland and Norris 2002, Emlen et al. 2003).

All the simulations start at time $\mathrm{t}=0$ with 15 eagle pairs at the breeding area and 15 floaters randomly distributed among the dispersal areas. Firstly, we ran 100 simulations (each of 100 years), the outputs of the model therefore resulting in 1 (floater annual mortality) $>2$ (synchroneous/asynchroneous variation in environmental conditions) $>100$ simulations. For each of them we obtained the values of the number of individuals and pairs for year. At the end of each series of 100 simulations, the model generates the means of number of individuals, number of pairs and productivity (fledged young) for the 100 years of each simulation.

Finally, we ran a second simulation in which we investigated the effect of increased floater mortality (e.g. occurrence of aleatory natural catastrophes, habitat destruction caused by human activities or building of new power lines in settlement areas) on population stability. Because the main interest of this simulation was to assess the effect of an increase in mortality within settlement areas on the breeding population, we did not separate the effects induced by environmental disaster and those caused by habitat destruction (Casagrandi and Gatto 2002). Understanding the influence of such types of disturbances is of paramount importance for conservation, because of their potentially severe impact on population stability (Casagrandi and Gatto 2002). To test the appearance of a disturbance event (i.e. accidental mortality), we set the floater mortality to $30 \%$. We then ran two simulations. In the first, the environmental stochasticity for breeders and floaters was correlated (synchroneous variation in environmental conditions; i.e. each year was of the same quality - poor or good - for both). In the second, it was not correlated (asynchroneous variation in environmental conditions; one year could be good for breeders and poor for floaters, or conversely). As for the first series of 100 simulations, the model generates a final output with the means of 
number of individuals, number of pairs and productivity. See Appendix C for additional information on the simulation parameters.

\section{Data analyses}

When data were not normally distributed, they were transformed. If normalization was not possible, we used nonparametric tests. In particular, we used the Kruskal Wallis test to address the relationship between $\mathrm{S}, \mathrm{N}$ and $\mathrm{M}$, as well as the possible changes in the parameters of the model outputs as the simulation progress, or in different situations of productivity, stochasticity and annual mortality. We employed the Mann-Whitney U-test to compare the patterns in the different population parameters (a) when the individuals were submitted to synchroneous or asynchroneous variation in environmental conditions, and (b) when, for the same situation of synchrony of environmental stochasticity, mortality in settlement areas varied from 5 to $30 \%$. All means are given with 9 sd, all tests are two-tailed, and statistical significance was set at pB0.05. Software package was SPSS 10.0 .

\section{Results}

Ratio "number of individuals : available settlement areas’’. Fixed numbers of floaters

Because the only possible modification in this situation was eagle death by starvation, the population only reaches a theoretical best condition of biological equilibrium when $S=1$, that is on the upper surface of the graph in Fig. 1. Actually, on the other regions of the graph, the forced saturation at the beginning of the simulation leads to high mortality (high as $\mathrm{N}$ is high), with the result that the final population is somewhat smaller than the population that could be maintained by the corresponding number of settlement areas. We can distinguish three well delimited zones on the graph surface: (1) the upper plane $(S=1)$ where the high number of $\mathrm{M}$ areas allows the $\mathrm{N}$ eagles to survive easily due to rabbit availability; (2) the lower part characterised by the lowest values of $\mathrm{S}$ and a gentle slope increasing as $\mathrm{M}$ augments. In this area the rabbit status does not allow the individuals to feed and, consequently, the population collapses and the few "survivors" are the individuals that remain when the death of the others enables them to feed; and (3) the transition slope, where $M$ does not allow the survival of all the original $\mathrm{N}$ but, after the death of several eagles, the population reaches stability. This zone is probably closer to the situation of natural populations.

These results allowed us to investigate the relationship between $\mathrm{S}, \mathrm{N}$ and $\mathrm{M}$. As shown in Fig. 2, for $\mathrm{S}=50 \%$ (a) and $80 \%$ (b), the number of individuals that can survive is highly correlated with the number of available settlement areas (for both 50 and 80\%: $r=0.99, p=0.0001$, $\mathrm{n}=18$ ), but the number of eagles that can survive does not change significantly $(\mathrm{t}=-0.764$, $\mathrm{df}=34, \mathrm{p}=0.45)$, although $\mathrm{N}_{80 \%} \mathrm{BN}_{50 \%}$. Using the values of $\mathrm{M}, \mathrm{N}$ and the corresponding survival $\mathrm{S}(\mathrm{M}, \mathrm{N})$, we calculated $S$ variation: $[S(M, N)-S(M-1, N)]$. We observed three results in relation to the survival increase for each value of $\mathrm{M}$ and $\mathrm{N}$ when the settlement areas availability increases by $M \neq 1$, (an approximation to the survival derivative as to the number of areas $\mathrm{dS} / \mathrm{dM}$ ). The first result is that $\mathrm{S}$ generally reaches the largest increase
Fig. 1. Output of the fixed simulation on the ratio number of individuals: available settlement areas. Number of settlement areas, number of individuals, and eagle survival (original population/final population) after time $=5$ years are plotted. The transition slope, between the upper plane (where the high number of areas allows all the eagle population to survive) and the lower part (where the low rabbit status leads to population collapse), better reflects the situation of natural populations in which the availability of settlement areas does not allow the survival of all the individuals but in which, after the death of several of them, population reaches stability.

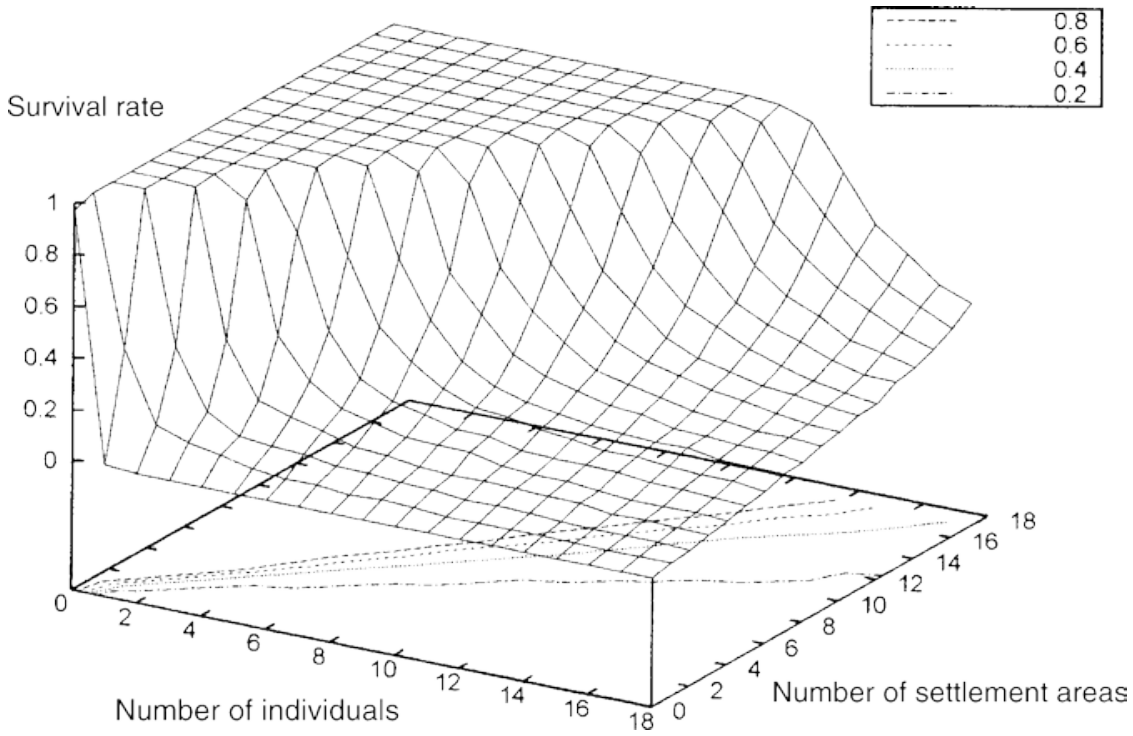


when, for a given number of floaters, the minimum increase in settlement areas is $\mathrm{M}=\mathrm{N} \neq 2$. The second result is that, although each $M \neq 1$ increase enhances the probability of survival, the increase in the number of dispersing individuals lowers and homogenises the values of mean survival increase: the maximum increment of $\mathrm{S}$ is $58 \%$ for 2 floaters and 4 available areas, whereas the lower values were detected for the proportions of 18:20 (5.72\%) and 19:20 (5.95\%). The third result is that such variations of $\mathrm{S}$ are significantly different in the different classes of $\mathrm{N}$ (range 1-19; $\mathrm{H}=$ 73.01, $\mathrm{p}=0.0001, \mathrm{n}=18 ; \mathrm{K}-\mathrm{W}$ test).

Ratio "number of individuals : available settlement areas'". The varying number of floater simulation

The number of floaters reaches stability in the settlement areas in a time dependent on the productivity of the population (Fig. 3a): the higher the productivity, the sooner equilibrium is attained. Moreover, the number of floaters increases with increased productivity, although in a nonlinear way due to the coincident increase of mortality (Fig. 3b). That is, the number of floaters in a population is a function of the productivity in the breeding territories $(\mathrm{r}=0.91, \mathrm{p}=0.0001, \mathrm{n}=38)$ and is limited by the availability of settlement areas, mediated by the rabbit behavioural changes. Number $(\mathrm{H}=$ 1085.36, $\mathrm{df}=4, \mathrm{p}=0.0001$; Kruskal - Wallis test) and mortality $(H=997.76, d f=4, p=0.0001)$ of floaters for the different values of productivity within the breeding territories were different. Interestingly, when investigating a large productivity range (from 1 to 39 fledglings
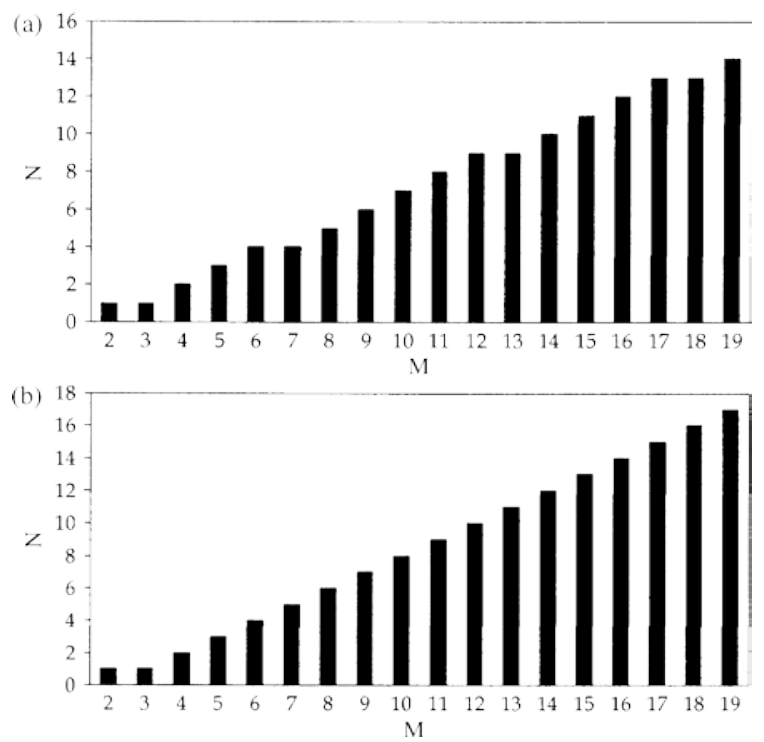

Fig. 2. Number of individuals (N) that can survive in $M$ settlement areas with a survival \80\% (A) and \50\% (B). Bars represent averages of 100 replicates. year ${ }^{-1}$ ), the number of floaters that can survive in the settlement areas is in a similar proportion (range approx. 1-3 individuals) to that shown by the fixed simulation, for which the highest values of $S$ corresponded $M=2-4$ times higher than $\mathrm{N}$.

Effects of synchronous and asynchronous variation in environmental conditions in settlement and breeding areas

Numbers of individuals and pairs in the breeding territories were significantly higher when the environmental conditions varied asynchronically (Fig. 4a, b; additional information in Appendix D). That is, negative events have less impact on breeder numbers when they take place in a temporally and spatially disjunct way (either in settlement or in breeding areas) and therefore affect only a portion of a population. Productivity was differently affected by the type of environmental stochasticity, being the same for both scenarios of probability of environmental stochasticity occurrence (Fig. 4c, Appendix D).

Effects of synchronous and asynchronous variation in environmental conditions in settlement and breeding areas when floater mortality increases

When we allowed floater mortality in settlement areas to increase (Fig. 4), significant negative changes occurred within each situation of environmental stochasticity and for both number of individuals (synchroneous variation in environmental conditions: $\mathrm{z}=-11.95, \mathrm{p}=0.0001$; asynchroneous variation in environmental conditions: $\mathrm{z}=$-11.93, $\mathrm{p}=0.0001$; Mann - Whitney U-test) and number of pairs (synchroneous variation in environmental conditions: $\mathrm{z}=-12.19, \mathrm{p}=0.0001$; asynchroneous variation in environmental conditions: $\mathrm{z}=$ $-12.16, \mathrm{p}=0.0001$ ).

As happened with floater mortality equal to $5 \%$, productivity (synchroneous variation in environmental conditions: $\mathrm{z}=-11.91, \mathrm{p}=0.0001$; asynchroneous variation in environmental conditions: $\mathrm{z}=-11.48, \mathrm{p}=$ 0.0001 ) was differently affected by the type of environmental stochasticity (Fig. 4c, Appendix D). Actually, productivity was influenced in the opposite way compared to numbers of individuals and pairs: (a) the higher the floater mortality, the higher the productivity; and (b) productivity was slightly higher when environmental stochasticity occurred synchronically. Because in our simulation breeding performance is a function of the saturation level of the population, such patterns reflect density-dependence. The buffer effect due to the absence of an upper threshold to population growth (i.e. large populations) is particularly obvious when we set floater 

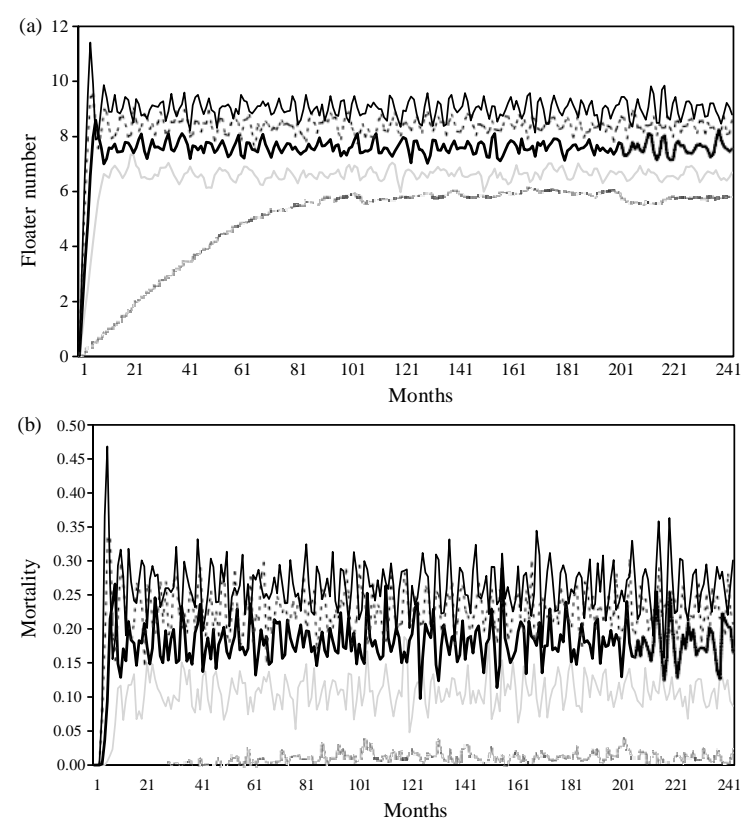

Fig. 3. The different ways in which productivity of the breeding population modulates the relationship between floaters and settlement areas. Number of floaters (a) and their mortality (b) within 10 settlement areas and with five different values of productivity by year in the breeding areas: 1 fledgling (soft line), 10 fledglings (grey line), 20 fledglings (bold black line), 30 fledglings (broken black line) and 39 fledglings (solid black line).

mortality at $30 \%$ : the population still comprises $>50$ eagles and $>10$ pairs.

Interestingly, for a high value of floater mortality, after the initial increase of productivity due to the density-dependent effects, a marked decrease in productivity appears as a feedback event, after approx. 20 years of relative stability (Fig. 4c).

\section{Discussion}

We showed that: (a) the survival of dispersing individuals (numbers of which depend on the productivity in breeding areas) is highly dependent on the number of available settlement areas, mediated by the time rabbits take to modify their behaviour to avoid predation; (b) environmental stochasticity has a stronger influence on the whole population (breeders floaters) when variations in environmental conditions occur in a synchroneous way, that is in both the settlement and breeding areas at the same time; (c) an increase of floater mortality within settlement areas leads to a negative impact on the stability and dynamics of breeders within the reproductive areas.

Although individual and population survival probabilities were evaluated as a function of several different factors, this is the first time, to our knowledge, that the relationship between survival, predator pressure, prey behaviour and patch availability (i.e. settlement areas) has been addressed. The novelty of our approach is the consideration of the eagle-rabbit game (or, in a more general context, the effect of predation pressure on prey behaviour). In a more general perspective, our results highlight the complexity and multiplicity of the effects mediated by the interaction between individuals and their environment. Factors influencing survival and predator-prey interactions should not only include prey density, its fluctuations, and the individual interactions with the environment (Jansen 2001), but also a change in prey behaviour that reduces its availability to
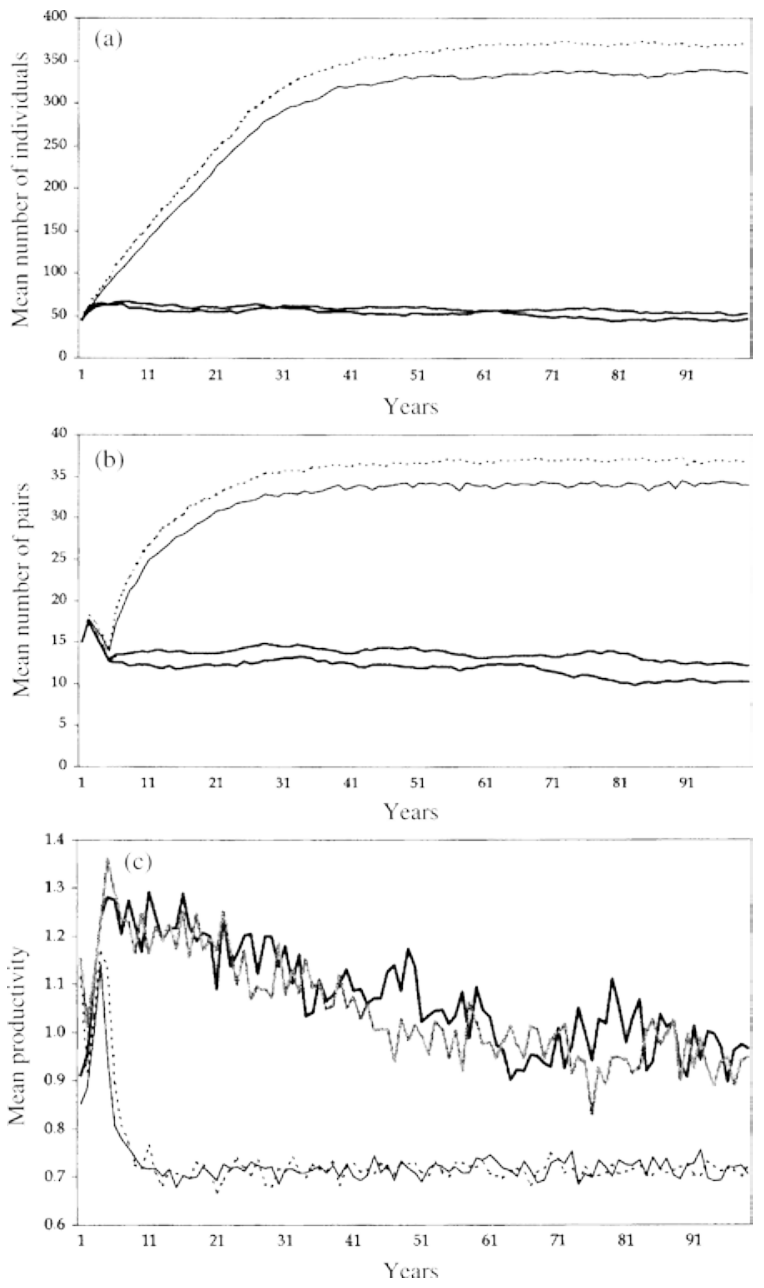

Fig. 4. Relationship between floater mortality $(5 \%=$ black lines; $30 \%=$ bold lines) in the settlement areas and mean patterns of number of individuals (a), number of pairs (b) and productivity (c) in the breeding areas for 100 simulations. Solid and broken lines represent patterns of synchroneous and asynchroneous occurrence of environmental stochastic events, respectively. Negative events such as environmental stochasticity affect populations (i.e. numbers of individuals and pairs) in a less substantial manner when they occur asynchronically between settlement and breeding areas. 
predators, even if the density significantly exceeds the predator needs. In this case, the number of areas needed for floater to survival is mainly a function of the time the prey takes to modify its behaviour. This type of dependence could explain why, starting at a specific threshold, an increase in the availability of settlement areas produced only slight changes in floater survival. As the number of individuals increases, the probability also increases that an eagle reaches an unexploited area, thereby starting the countdown to rabbit behavioural modification, with the consequence that a diffuse disturbance of the whole system of settlement areas could drastically reduce the availability of "unexploited" areas.

Our simulations highlight the link between the dynamics within settlement and breeding areas. Factors affecting floater survival also influenced the whole population persistence, which is in agreement with earlier theoretical and empirical works (Klomp and Furness 1992, Ruxton et al. 1997, Casagrandi and Gatto 2002, Etienne et al. 2002).

When mortality in the settlement areas increases, the whole number of individuals and breeding pairs in the breeding territories significantly decreases, especially when environmental stochasticity occurs synchronically in settlement and breeding areas. In a situation of low floater mortality, the demographic contribution of dispersers compensates for adult mortality in the breeding areas, which reduces extinction risk in the breeding segment of the population. Such an effect could be considered analogous to the "rescue effect" (Brown and Kodric-Brown 1977). In fact, when considering the scenario of a large population characterised by the absence of a threshold for a population size, both individual and pair numbers decreased by approx. five times when mortality increased from 5 to $30 \%$. In small populations, the effects can be more drastic, availability of a huge floater stock efficiently reducing local extinction risk by dampening stochastic fluctuations (Ferrer et al. 2004). Although dispersal rescue is generally predicted to be most advantageous to population persistence when there is little regional stochasticity that not affect adult survival (Hanski 1991), our results show the need to consider whether the variations in environmental conditions are synchronized or not in locations used by different sectors of the population when analysing its effects on population dynamics.

Many processes influencing population dynamics have their origin in local processes acting on subunits of the whole population (Harding and McNamara 2002), and several of them can be linked to the way dispersal affects demography in breeding territories, showing a profound impact on the whole population persistence. Therefore, a population can be viewed as a stratified system divided into a network of smaller subunits (e.g. dispersers and breeders, or settlement and breeding areas), the persis- tence of which is not only dependent on the local dynamics within each breeding unit, but also on the probability that floaters survive dispersal each year and are integrated into the reproductive portion of the population, relative to the number of individuals that die in both settlement and breeding areas. Danchin and Cam (2002) have already pointed out, albeit in a different context (i.e. studies of reproductive tradeoffs), that nonbreeding individuals need to be taken more into account in population studies. In the same way that population dynamics have a strong influence on dispersal (Murrell et al. 2002), it is crucial to consider floater dynamics when modelling population dynamics, since it is obvious that there is a link between the settlement and breeding area dynamics, which influences the whole population. Because productivity is densitydependent, this parameter was shown to be less sensitive at the beginning of an increase in floater mortality (reducing the saturation of the breeding population), almost up to a critical threshold (the $30 \%$ we set in the simulation to test the effects of mortality increase in settlement areas on breeding territories). In fact, productivity initially increases with a fall in saturation brought about by a decline in the number of pairs, only to fall later. Such results highlight the importance of density dependence to any understanding of population ecology, giving it a central place in simulation studies, as underlined by Sutherland and Norris (2002).

Our results showed that population studies have to make assumptions about whether and how demographic parameters in breeding areas are dependent on mortality in settlement areas, in a similar way to that which occurs with source-sink dynamics (Gundersen et al. 2001). Generally, because the areas where dispersers settle in the dispersal stage are unknown, fewer efforts are devoted to conservation of these sites than to breeding territories, which results in less effective conservation. Moreover, as in our study species, dispersers frequently use areas in which high levels of anthropogenic disturbance result in high mortality rates ("sink" areas). This might have important biological consequences; for example, conservation efforts such as protection of the breeding areas or nesting sites in a threatened population can be ineffective if the real problem is in the settlement areas. In most conservation programmes efforts are concentrated on breeding areas. Our results highlight the importance for the stability of a population of a strong knowledge of the location and number of the areas used by dispersers, as well as the mechanisms regulating floater survival. We have already demonstrated the importance of constantly checking the status and trends of the juvenile portion of the population in order to detect the population decline early (Ferrer et al. 2003).

Conservation theory is often concerned with the probability that a stochastic event occurs within a 
population and, therefore, with the extinction risk in a (meta)population subject to it (Tillman et al. 1994, Hill and Caswell 2001). Generally, simulations of the relationship between increased stochasticity and population response generate unambiguous results proving that the positivity of the sign of the relationship between stochasticity and extinction risk is a robust property, valid for a broad class of population models (Alvarez 2001). Our simulation has added the new information that population dynamics differ when the stochastic event occurs in a synchroneous or asynchroneous way in breeding and settlement areas. The details of how stochasticity operates are then crucial to questions of population equilibrium and persistence (Lande et al. 1998). Interestingly, Johst and Drechsler (2003) obtained similar results in a spatial context, showing that spatially correlated environmental stochasticity reduced metapopulation persistence.

Our model outputs involve additional implications relevant to population management and conservation plans. They help us to understand, detect and predict population responses to environmental stochasticity. For example, reintroduction and/or restocking of species could have a higher probability of success if they took place simultaneously in different locations, where environmental conditions vary asynchronically, which would minimize the possibility that largescale fluctuations affect all the portions of a population in a highly correlated way (Harrison and Quinn 1989).

Acknowledgements - This paper has greatly benefited from conversations and comments by J. Bascompte and C. Melián. During this research, V.P. has been supported by a Marie Curie Fellowship of the European Community programme "Improving the Human Research Potential and the SocioEconomic Knowledge Base" under contract number HPMFCT-2000-01098. The author is solely responsible for information communicated and the European Commission is not responsible for any view or resulted expressed. The Junta de Andalucía also contributed to the financial support of some parts of this project.

\section{References}

Acosta, C. A. 2002. Spatially explicit dispersal dynamics and equilibrium population sizes in marine harvest refuges. - ICES J. Mar. Sci. 59: 458-468.

Alvarez, L. H. R. 2001. Does increased stochasticity speed up extinction? - J. Math. Biol. 43: 534-544.

Bascompte, J., Possingham, H. and Roughgarden, J. 2002. Patchy populations in stochastic environments: critical number of patches for persistence. - Am. Nat. 159: 128137.

Begon, M., Harper, J. L. and Townsend, C. R. 1996. Ecology: individuals, populations and communities. - Blackwell Science Ltd.

Brown, J. H. and Kodric-Brown, A. 1977. Turnover rates in insular biogeography: effect of immigration on extinction. - Ecology 58: 445-449.

Casagrandi, R. and Gatto, M. 2002. Habitat destruction, environmental catastrophes, and metapopulation extinction. - Theor. Popul. Biol. 61: 127-140.
Clobert, J., Danchin, E., Dhondt, A. A. et al. 2001. Dispersal. - Oxford Univ. Press

Danchin, E. and Cam, E. 2002. Can nonbreeding be a cost of breeding dispersal? - Behav. Ecol. Sociobiol. 51: 153-163.

DeAngelis, D. L. and Gross, L. J. (eds). 1992. Individual-based models and approaches in ecology. - Chapman and Hall.

Emlen, J. M., Freeman, D. C., Kirchhoff, M. D. et al. 2003. Fitting population models from field data. - Ecol. Model. 162: $119-143$.

Etienne, R., Wertheim, B., Hemerik, L. et al. 2002. The interaction between dispersal, the Allee effect and scramble competition affects population dynamics. - Ecol. Model. 148: $153-168$.

Ferrer, M. 1992. Regulation of the period of postfledging dependence in the Spanish imperial eagle Aquila adalberti . - Ibis 134: 128-133.

Ferrer, M. 1993a. Reduction in hunting success and settlement strategies in young Spanish imperial eagles. - Anim. Behav. 45: 406-408.

Ferrer, M. 1993b. Juvenile dispersal behaviour and natal philopatry of a long-lived raptor, the Spanish imperial eagle Aquila adalberti . - Ibis 135: 132-138.

Ferrer, M. 2001. The Spanish imperial eagle. - Lynx Edicions.

Ferrer, M. and Calderón, J. 1990. The Spanish imperial eagle Aquila adalberti in Doñana National Park: a study of population dynamics. - Biol. Cons. 51: 151-161.

Ferrer, M. and Dobado-Berrios, P. 1998. Factors affecting plasma chemistry values of the Spanish imperial eagle, Aquila adalberti . - Comp. Biochem. Phys. A 120: 209-217.

Ferrer, M. and Bisson, I. 2003. Age and territory quality effects on fecundity in the Spanish imperial eagle. - Auk 120: 180186.

Ferrer, M. and Penteriani, V. 2003. A process of pair formation leading to assortative mating: passive age-assortative mating by habitat heterogeneity. - Anim. Behav. 66: 137-143.

Ferrer, M., Penteriani, V., Balbontín, J. et al. 2003. The proportion of immature breeders as a reliable early warning signal of population decline: evidence from the Spanish

Imperial Eagle in Doñ ana. - Biol. Cons. 114: 463-466. Ferrer, M., Otalora, F. and García-Ruiz, M. 2004. Densitydependent age of first reproduction as a buffer affecting persistence of small populations. - Ecol. Appl. 14: 616-624.

Flather, C. H. and Bevers, M. 2002. Patchy reaction-diffusion and population abundance: the relative importance of habitat amount and arrangement. - Am. Nat. 159: 40-56.

Gamarra, J. G. P. and Solé, R. V. 2000. Bifurcations and chaos in ecology: lynx returns revisited. - Ecol. Lett. 3: 114-121.

Gundersen, G., Johannesen, E., Andreassen, H. P. et al. 2001. Source-sink dynamics: how sinks affect demography of sources. - Ecol. Lett. 4: 14-21.

Hanski, I. 1991. Single-species metapopulation dynamics: concepts, models, and observations. - Biol. J. Linn. Soc. 42: $17-38$.

Harding, K. C. and McNamara, J. M. 2002. A unifying framework for metapopulation dynamics. - Am. Nat. 160: 173-185.

Harrison, S. and Quinn, J. F. 1989. Correlated environments and the persistence of metapopulations. - Oikos 56: 293298.

Hill, M. F. and Caswell, H. 2001. The effects of habitat destruction in finite landscapes: a chain-binomial metapopulation model. - Oikos 93: 321-331.

Hill, M. F., Hastings, A. and Botsford, L. W. 2002. The effects of small dispersal rates on extinction times in structured metapopulation models. - Am. Nat. 160: 389-402.

Jansen, V. A. A. 2001. The dynamics of two diffusely coupled predator-prey populations. - Theor. Popul. Biol. 59: 119131.

Johst, K. and Drechsler, M. 2003. Are spatially correlated or uncorrelated disturbance regimes better for the survival of species? - Oikos 103: 449-456.

Klomp, N. I. and Furness, R. W. 1992. Non-breeders as a buffer against environmental stress: declines in numbers of 
great skuas on Foula, Shetland, and prediction of future recruitment. - J. Appl. Ecol. 29: 341-348.

Lande, R., Engen, S. and Sæther, B.-E. 1998. Extinction times in finite metapopulation models with stochastic local dynamics. - Oikos 83: 383-389.

Murrell, D. J., Travis, J. M. J. and Dytham, C. 2002. The evolution of dispersal distance in spatially-structured populations. - Oikos 97: 229-236.

Nathan, R. 2001. The challenges of studying dispersal. - Trends Ecol. Evol. 16: 481-483.

Paradis, E., Baillie, S. R. and Sutherland, W. J. 2002. Modeling large-scale dispersal distances. - Ecol. Model. 151: 279-292

Penteriani, V., Gallardo, M. and Roche, P. 2002. Landscape structure and food supply affect eagle owl (Bubo bubo) density and breeding performance: a case of intra-population heterogeneity. - J. Zool. (London) 257: 365-372.

Ruxton, G. D., Gonzalez-Andujar, J. L. and Perry, J. N. 1997. Mortality during dispersal and the stability of a metapopulation. - J. Theor. Biol. 186: 389-396.

Sæther, B.-E., Steinar, E., Lande, R. et al. 2002. Density dependence and stochastic variation in a newly established population of a small songbird. - Oikos 99: 331-337.

Sutherland, W. J. and Norris, K. 2002. Behavioural models of population growth rates: implications for conservation and prediction. - Philos. Trans. R. Soc. B 357: 1273-1284.

Tillman, D., May, R. M., Lehman, C. L. et al. 1994. Habitat destruction and the extinction debt. - Nature 371: 65-66.

Walters, J. R. 2000. Dispersal behavior: an ornithological frontier. - Condor 102: 479-481.

Yáber, M. C. and Rabenold, K. N. 2002. Effects of sociality on short-distance, female-biased dispersal in tropical wrens. - J. Anim. Ecol. 71: 1042-1055.

\section{Appendix A}

A population of $\mathrm{N}$ predators distributed in $\mathrm{M}$ distinct settlement areas is simulated. A description of the behaviour of the system follows describing the events affecting to just one of the predators, the jth $(0 \mathrm{Bj} 5 \mathrm{~N})$ individual living at time $t$ in the ith $(0 \mathrm{BI} 5 \mathrm{M})$ area. The whole model is built-up by iterative evaluation (on a daily basis) of the described equations for each predator (see pseudo-code at the end of this appendix).

The ith settlement area is characterized by a number of predators $n_{I}\left(S_{i=1}^{M} n_{i}=N\right)$ and a "prey state of alert", $a_{i}$. This value is updated daily as a function of the presence of predators in the area,

$\mathrm{a}_{\mathrm{i}}(\mathrm{t} \neq 1)=\mathrm{a}_{\mathrm{i}}(\mathrm{t}) \neq \mathrm{K}_{\mathrm{i}}(\mathrm{t}) \mathrm{da}$

da being a constant value adjusted on the basis of actual data from the Doñ ana population ( $\mathrm{da} \div 0.3)$ and $\mathrm{Ki}$ a discrete boolean function valued $\mathrm{Ki}=1$ if $\mathrm{n}_{\mathrm{i}}>0$ and $\mathrm{Ki}=-1$ if $\mathrm{n}_{\mathrm{i}}=0$. To avoid unrealistic memory effects in extreme cases in which an area stays for a very long time either occupied or unoccupied, two limiting values for $a_{i}$ are defined so that

$0: 1=\min _{\mathrm{a}} 5 \mathrm{a}_{\mathrm{i}}(\mathrm{t}) 5 \max _{\mathrm{a}}=0: 96 \mathrm{t}$

The probability $\mathrm{H}_{\mathrm{i}}$ of a predator living in this area hunting a prey a given day is modelled as a Boltzmann distribution

$\mathrm{H}_{\mathrm{i}}=\mathrm{A}_{2} \neq\left(\mathrm{A}_{1}-\mathrm{A}_{2}\right)=1 \neq \exp \left(\mathrm{a}_{\mathrm{i}}-\mathrm{x}_{0}\right)=\mathrm{dx}$

where $A_{1} \div 1$ and $A_{2} \div 0$ are respectively the asymptotic maximum and minimum values, $x_{0}$ is the centre of the distribution (i.e. $\mathrm{H}_{\mathrm{i}}\left(\mathrm{x}_{0}\right)=1 / 2$ ) and $\mathrm{dx}$ is a reciprocal scale factor for the abscissa, hence controlling the slope of the transition from $A_{1}$ to $A_{2}$.

The jth predator is characterized by a "damage state", $\mathrm{d}_{\mathrm{j}}$ that is updated daily as

$\mathrm{d}_{\mathrm{j}}(\mathrm{t} \neq 1)=\mathrm{d}_{\mathrm{j}}(\mathrm{t}) \neq \mathrm{S}_{\mathrm{j}}(\mathrm{t}) \mathrm{fu}-\mathrm{dc}$

fu being a constant value representing the "food unit", (how much "energy" there is in a prey) adjusted on the basis of actual data as $\mathrm{fu} \div 3 \mathrm{dc}$, where dc is the "day consumption", i.e. the amount of "energy" needed to survive one day). $\mathrm{S}_{\mathrm{j}}$ is a discrete boolean function valued $\mathrm{S}_{\mathrm{j}}=-1$ if the predator hunts successfully and $\mathrm{S}_{\mathrm{j}}=0$ else. A successful hunting event means $\mathrm{H}_{\mathrm{i}}(\mathrm{t}) \mathrm{BR}$, $\mathrm{i}$ being the area where the jth predator lives and $05 \mathrm{R} B 1$ being a pseudo-random uniform deviate. $d_{j}$ is also bounded as in Eq. 2 to be more than 0.05 . There is no explicit upper level for $d_{j}$ as both death and moving to another area are triggered by high values of this status variable.

The death and move probabilities are modelled using Boltzmann probability distributions

$\mathrm{T}_{\mathrm{j}}=\mathrm{A}_{2} \neq\left(\mathrm{A}_{1}-\mathrm{A}_{2}\right)=1 \neq \exp \left(\mathrm{d}_{\mathrm{j}}-\mathrm{x}_{0}\right)=\mathrm{dx}$

If $\mathrm{T}_{\mathrm{j}}$ Bst the predator moves to a different area randomly chosen among the M-1 areas available and distinct from the current one. st is an adjustable parameter indicating the "warning level"' below which the predator really needs to look for food elsewhere and has been adjusted to st $\div 0.3$ on the basis of observed moving frequencies. Eventually, $\mathrm{T}_{\mathrm{j}}$ may fall below a more restrictive threshold dy $\div 0.1$. At this level the predator dies.

The following pseudo-code shows the overall simulation: set Boltzmann distribution (A1, A2, x0, dx) set adjustable parameters ( $N, M$, da, dc, st, dy)

for $\mathrm{i}=1$ to $\mathrm{M}$ \{

$$
\text { setup ith area }(\mathrm{a}[\mathrm{i}]=\text { random })
$$

\}

for $\mathrm{j}=1$ to $\mathrm{N}$ \{

setup $\mathrm{jth}$ predator $(\mathrm{d}[\mathrm{j}]=$ random $)$

link predator $\mathrm{j}$ to a random area

\}

for $\mathrm{t}=1$ to time_limit \{

for $\mathrm{i}=1$ to $\mathrm{M}$ \{

update area i (Eq. 1)

\}

for $\mathrm{j}=1$ to $\mathrm{N}$ \{

update predator j (Eq. 4)

if $T[j]$ Bdy \{

kill the predator

\}

else if $\mathrm{T}[\mathrm{j}]$ Bst \{

move the predator to a different area

\} 


\section{Appendix B}

The eagle-rabbit game in a simple situation with 1 eagle and 2 settlement areas during 90 days. The eagle status corresponds to the individual physiological condition (see model characteristics), whereas the zone status corresponds to the likelihood of catching a rabbit in the area. Vertical lines mark the changes between the settlement areas (starting with the zone 0 ).

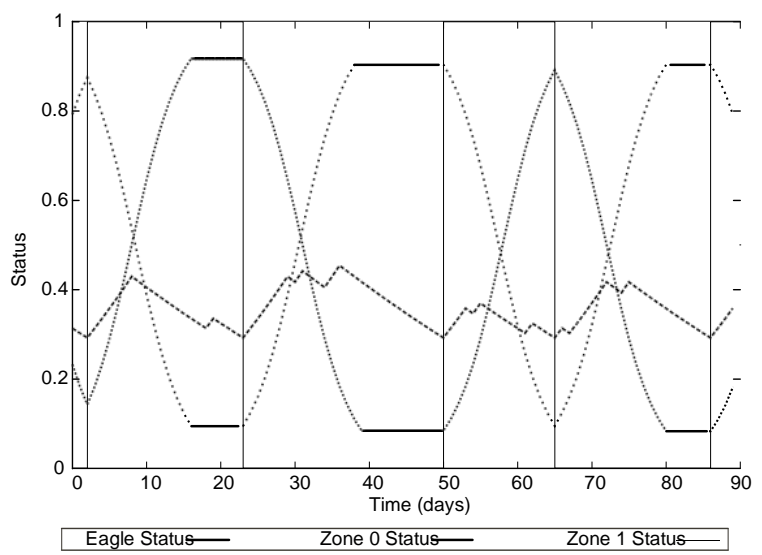

The simplicity of this case (1 eagle and 2 settlement areas over 90 days) allows us to better represent the evolution of the basic process without the complications due to all the possible interactions between all the individuals present in the same area at the same time. The figure shows that: (a) the probability of obtaining food in each area decreases as soon as the predator arrives, with a range of values between 0.9 and 0.1 over approx. 12 days; (b) in the area where the eagle is absent the pattern is exactly the opposite; (c) the health condition of the predator increases when it obtains a prey and decreases in the days of fasting; (d) because in this simple simulation we have only one eagle moving between two areas, the new area the predator moves to (vertical lines) will always be in a condition of low rabbit state of alert and the probability of hunting successfully will consequently be high, allowing the individual to improve its physical condition rapidly. This situation is not the rule when there is more than one eagle; the probability of successful hunting (for the same number of available settlement areas) then decreases with the increase in the number of dispersers. Because floater movements are mainly determined by the behavioural alteration of their prey, and do not derive from specific characteristics of this eagle species, the models can be considered as a broad simulation of general predator dynamics during their juvenile dispersal.

\section{Appendix C}

Eight constant parameters were used to simulate the effects of the synchronous and asynchronous variations of environmental conditions in settlement and breeding areas:

(a) Death timing - The fecundity of a pair, and independently for the species, depends on the time of the year in which mortality occurs, especially in species characterized by a long breeding cycle (Penteriani, Otalora and Ferrer, unpubl.). For this reason, two different temporal sequences were selected at random in the simulation: $75 \%$ of the time we ran the sequence mating Odeath Oreproduction and 25\% of the time the sequence death Omating Oreproduction. Obviously, the first sequence results in productivity $=0$;

(b) Productivity - Density-dependent, relying on the population saturation. For a saturation threshold $=1$ (population totally saturated), the probability of producing $0,1,2$ or 3 young is $47.62 \%, 38.10 \%, 9.52 \%$, and $4.76 \%$, respectively. For a saturation threshold $=0$, the probability is $20 \%, 30 \%, 30 \%$, and $20 \%$. For intermediate values of saturation, this probability is calculated as the weighted average of the extreme values, i.e. saturation $>$ productivity $\quad(1) \$(1$-saturation $)>$ productivity (0);

(c) Mortality - The mortality is calculated as the combination of two distributions of probability: (1) natural mortality, which precludes the possibility of an individual living indefinitely if the other parameters of the model correspond to a scenario with increased survival. Natural mortality is represented as a sigmoidal distribution centred on 30.0 and with exponent 10.0; and (2) accidental mortality, corresponding to a probability distribution with modal values of $60 \%$ (mortality for the first year of life), $6 \%$ (mortality of mated individuals), and a value of $5 \%$ for nonmated eagles $>1$ year old. This latter value was set to $30 \%$ in the second series of simulations, when evaluating the influence of increased floater mortality due to anthropogenic disturbance (e.g. habitat destruction, electrocution). The environmental stochasticity is represented as a normal stochastic variable of mean 1.0 and standard deviation 1.0 that multiplies the probability of accidental death and changes from year to year;

(d) Sexual maturity - The age at first breeding was set at 3 years old;

(e) Saturation - The saturation of the breeding territories, dependent on breeding population characteristics, is represented by a sigmoidal distribution centred on 30.0 and with exponent 10.0;

(f) Sex ratio - Equiprobable, that is $50 \%$ of the offspring are males and $50 \%$ are females;

(e) Mating probabilities - The probability that two non-mated, sexually mature and different-sex individuals become a pair is density-dependent and corresponds to 1-saturation. 
Shuffle: allows aleatory rearrangement of the list of living individuals in each new simulation, which avoids possible uncontrolled correlations between individuals. There would otherwise be a particular risk of age related correlations because, after a few years, individuals end up roughly sorted by age as new chicks are added to the end of the list of individuals. This introduces a bias in all density-dependent processes, for example mating, as the simulated events are evaluated iteratively over the list. Simulations were run with the Delphi (object-oriented) programming language.

\section{Appendix D}

Relationship between floater mortality in the settlement areas and number of individuals, number of pairs and productivity in the breeding areas. The means of such parameters for 100 simulations are compared (Mann Whitney U-test) between synchroneous and asynchroneous occurrence of environmental stochasticity, for values of floater mortality of 5 and 30\%. Numbers of individuals and pairs in the breeding territories were significantly higher when the environmental stochasticity occurred asynchronically within settlement and breeding areas. Productivity seemed to be less affected by the occurrence of environmental stochasticity.

\begin{tabular}{|c|c|c|c|c|}
\hline & \multicolumn{2}{|c|}{$\begin{array}{l}\text { Synchroneous vs } \\
\text { asynchroneous }\end{array}$} & \multirow[t]{2}{*}{$\mathrm{z}$} & \multirow[t]{2}{*}{$\mathrm{p}$} \\
\hline & $\bar{x} \mathbf{9 s d}$ & $\bar{x}$ 9sd & & \\
\hline \multicolumn{5}{|l|}{ Floater mortality $=5 \%$} \\
\hline Number of individuals & 283.4981 .7 & 311.5989 .8 & -5.59 & 0.0001 \\
\hline Number of pairs & 31.595 .0 & 34.195 .5 & -7.42 & 0.0001 \\
\hline Productivity & 0.790 .0 & 0.797 .7 & -0.62 & 0.533 \\
\hline \multicolumn{5}{|l|}{ Floater mortality $=30 \%$} \\
\hline Number of individuals & 52.595 .1 & 58.593 .7 & -7.96 & 0.0001 \\
\hline Number of pairs & 11.991 .2 & 13.790 .8 & -10.54 & 0.0001 \\
\hline Productivity & 1.190 .1 & 1.090 .1 & -1.87 & 0.061 \\
\hline
\end{tabular}

